\title{
Four-Month Moxifloxacin-Based Regimens for Drug-Sensitive Tuberculosis
}

\author{
Stephen H. Gillespie, M.D., D.Sc., Angela M. Crook, Ph.D., \\ Timothy D. McHugh, Ph.D., Carl M. Mendel, M.D., Sarah K. Meredith, M.B., B.S., \\ Stephen R. Murray, M.D., Ph.D., Frances Pappas, M.A., Patrick P.J. Phillips, Ph.D., \\ and Andrew J. Nunn, M.Sc., for the REMoxTB Consortium*
}

ABSTRACT

\section{BACKGROUND}

Early-phase and preclinical studies suggest that moxifloxacin-containing regimens could allow for effective 4-month treatment of uncomplicated, smear-positive pulmonary tuberculosis.

\section{METHODS}

We conducted a randomized, double-blind, placebo-controlled, phase 3 trial to test the noninferiority of two moxifloxacin-containing regimens as compared with a control regimen. One group of patients received isoniazid, rifampin, pyrazinamide, and ethambutol for 8 weeks, followed by 18 weeks of isoniazid and rifampin (control group). In the second group, we replaced ethambutol with moxifloxacin for 17 weeks, followed by 9 weeks of placebo (isoniazid group), and in the third group, we replaced isoniazid with moxifloxacin for 17 weeks, followed by 9 weeks of placebo (ethambutol group). The primary end point was treatment failure or relapse within 18 months after randomization.

\section{RESULTS}

Of the 1931 patients who underwent randomization, in the per-protocol analysis, a favorable outcome was reported in fewer patients in the isoniazid group (85\%) and the ethambutol group ( $80 \%$ ) than in the control group (92\%), for a difference favoring the control group of 6.1 percentage points $(97.5 \%$ confidence interval [CI], 1.7 to 10.5) versus the isoniazid group and 11.4 percentage points (97.5\% CI, 6.7 to 16.1 ) versus the ethambutol group. Results were consistent in the modified intention-totreat analysis and all sensitivity analyses. The hazard ratios for the time to culture negativity in both solid and liquid mediums for the isoniazid and ethambutol groups, as compared with the control group, ranged from 1.17 to 1.25 , indicating a shorter duration, with the lower bounds of the $95 \%$ confidence intervals exceeding 1.00 in all cases. There was no significant difference in the incidence of grade 3 or 4 adverse events, with events reported in 127 patients (19\%) in the isoniazid group, 111 (17\%) in the ethambutol group, and 123 (19\%) in the control group.

\section{CONCLUSIONS}

The two moxifloxacin-containing regimens produced a more rapid initial decline in bacterial load, as compared with the control group. However, noninferiority for these regimens was not shown, which indicates that shortening treatment to 4 months was not effective in this setting. (Funded by the Global Alliance for TB Drug Development and others; REMoxTB ClinicalTrials.gov number, NCT00864383.)
From the University of St. Andrews Medical School, St. Andrews (S.H.G.), and the Medical Research Council Clinical Trials Unit at University College London (A.M.C., S.K.M., P.P.J.P., A.J.N.) and the Division of Infection and Immunity, University College London (T.D.M.), London - both in the United Kingdom; and the TB Alliance, New York (C.M.M., S.R.M., F.P.). Address reprint requests to Dr. Gillespie at the School of Medicine, University of St. Andrews, North Haugh, St. Andrews KY16 9TF, United Kingdom, or atshg3@st-andrews.ac.uk.

*A complete list of investigators and committee members in the Rapid Evaluation of Moxifloxacin in Tuberculosis (REMoxTB) study is provided in the Supplementary Appendix, available at NEJM.org.

This article was published on September 7, 2014, at NEJM.org.

DOI: 10.1056/NEJMoa1407426 Coppright @ 2014 Massachusetts Medical Society. 
SHORT-TERM TUBERCULOSIS TREATment regimen could improve rates of adherence, reduce rates of adverse events, and lower costs. Fluoroquinolones have shown promising activity against mycobacteria ${ }^{1}$ and are established as a critical component of the treatment of multidrug-resistant tuberculosis, ${ }^{2,3}$ with later fluoroquinolones recognized as having a more potent effect. It has been proposed that these drugs may have a role in reducing the duration of tuberculosis treatment. ${ }^{4}$

Moxifloxacin has been approved for a range of indications globally. ${ }^{5}$ It has favorable pharmacokinetics, a large volume of distribution, and penetration into epithelial-lining fluid and macrophages. ${ }^{6-8}$ The activity of moxifloxacin in vitro against Mycobacterium tuberculosis, which has been confirmed in murine models ${ }^{9}$ and in clinical monotherapy studies, ${ }^{10,11}$ has raised the prospect that the drug could be used as part of an improved regimen. ${ }^{1}$ Subsequent studies in mice showed that combination regimens that included moxifloxacin had greater bactericidal activity than standard treatment and could produce cure without relapse after a shorter treatment duration. ${ }^{12,13}$

When different fluoroquinolones were substituted for ethambutol in a clinical trial, the moxifloxacin-containing regimen produced the most rapid decline in bacterial load and in the proportion of patients with culture negativity at 8 weeks. ${ }^{14}$ These findings were confirmed by investigators in Brazil. ${ }^{15}$ In contrast, substituting moxifloxacin for isoniazid in an 8-week study resulted in a nonsignificant enhancement in bactericidal effect. ${ }^{16}$

On the basis of supportive evidence from phase 2 studies and the uncertain relationships between 8-week bacteriologic data and the duration of effective therapy, we designed the Rapid Evaluation of Moxifloxacin in Tuberculosis (REMoxTB) study to determine whether the replacement of either isoniazid or ethambutol with moxifloxacin would provide effective tuberculosis treatment in 4 months, as compared with the standard 6-month regimen.

\section{METHODS}

\section{STUDY DESIGN AND OVERSIGHT}

REMoxTB was a placebo-controlled, randomized, double-blind, phase 3 trial to test the noninferiority of two moxifloxacin-containing 4-month regimens, as compared with the standard 6-month regimen (Fig. S1 in the Supplementary Appendix, available with the full text of this article at NEJM. org). The full trial protocol and statistical analysis plan are also available at NEJM.org.

A trial steering committee with an independent chair supervised the conduct of the trial. An independent data and safety monitoring committee with access to unblinded data oversaw the safety of the study patients. The ethics committee at University College London and all national and local ethics committees approved the study. The Food and Drug Administration, the Federal Institute for Drugs and Medical Devices (Bundesinstitut für Arzneimittel und Medizinprodukte), and the national regulatory authorities of the countries in which the trial was conducted reviewed and approved the protocol.

Bayer Healthcare donated moxifloxacin, and Sanofi donated rifampin. Neither company had any role in the study design, data accrual, data analysis, or manuscript preparation. Representatives of Bayer Healthcare reviewed the manuscript but did not suggest revisions. All the authors vouch for the completeness and accuracy of the data and analyses presented.

\section{STUDY PATIENTS}

Patients were adults ( $\geq 18$ years of age) who had newly diagnosed, previously untreated M. tuberculosis infection, as determined by positive results on sputum smears on two occasions, with culture-confirmed susceptibility to rifampin and fluoroquinolones. Patients who were coinfected with the human immunodeficiency virus (HIV) were eligible to participate in the study if the CD4+ count was at least 250 cells per cubic millimeter and they were not already receiving antiretroviral therapy. Detailed inclusion and exclusion criteria are provided in the Supplementary Appendix. All patients provided written or witnessed oral informed consent.

\section{RANDOMIZATION AND STUDY TREATMENTS}

Randomization was performed with the use of lists with blocks of variable sizes that were stratified according to the patient weight group and study center. During randomization, patients were assigned a unique study number selected sequentially from the appropriate randomization list that corresponded to the treatment pack allocated. Eligible patients were assigned in a 1:1:1 ratio to one of the following daily regimens: a control regimen, 
which consisted of isoniazid, rifampin, pyrazinamide, and ethambutol for 8 weeks, followed by 18 weeks of isoniazid and rifampin (control group); a regimen in which we replaced ethambutol with moxifloxacin for 17 weeks, followed by 9 weeks of placebo (isoniazid group); and a regimen in which we replaced isoniazid with moxifloxacin for 17 weeks, followed by 9 weeks of placebo (ethambutol group). Details about the regimens are provided in Figure S1 in the Supplementary Appendix.

In all three groups, drug doses were adjusted according to patient weight, as described in Table S2 in the Supplementary Appendix. Only statisticians who were responsible for preparing the reports for the independent data and safety monitoring committee and essential manufacturing and distribution staff members had access to the list of identifiers matched to the intervention.

\section{STUDY PROCEDURES}

After initial screening and baseline visits, patients were scheduled for eight weekly visits, which were followed by eight visits until 18 months after randomization (Fig. S1 in the Supplementary Appendix). All patients underwent a baseline clinical examination that included posteroanterior chest radiography, pregnancy testing if relevant, collection of two sputum specimens for microbiologic examination, physical examination, tests of visual acuity (Ishihara and Snellen), and urinalysis. Safety monitoring - which included testing of hepatic function (aspartate aminotransferase, alanine aminotransferase, and bilirubin), vitamin $\mathrm{K}$, prothrombin time, partial thromboplastin time, blood count (hemoglobin and platelet count), urea, electrolytes, and creatinine - was performed at screening and at weeks 2, 8, 12, and 17 , with additional liver-function testing at week 4.

Sputum was decontaminated with acetylcysteine-sodium hydroxide, examined microscopically, and cultured on Lowenstein-Jensen solid medium and in liquid medium in a Mycobacteria Growth Indicator Tube (MGIT) (Becton Dickinson). All analyses were performed according to the REMoxTB laboratory and quality manuals (available on request). We performed mycobacterial speciation using the AccuProbe assay (GenProbe), and determined the susceptibility of strains to streptomycin, isoniazid, rifampin, and pyrazinamide using the MGIT manufacturer's instruc- tions. We tested the susceptibility to moxifloxacin using a breakpoint of $0.125 \mathrm{mg}$ per liter. In countries with a high rate of multidrug-resistant tuberculosis or quinolone resistance ( $>5 \%$ ), initial sputum samples were tested for rifampin resistance with the use of the GenoType MTBDRplus assay and GenoType MTBDRsl assay, respectively (Hain Lifescience). We used 24-locus mycobacterial-interspersed-repetitive-unit (MIRU) analysis to compare the initial strains with the recurrence strains. ${ }^{17}$

\section{STUDY OUTCOMES}

The primary efficacy outcome was the proportion of patients who had bacteriologically or clinically defined failure or relapse within 18 months after randomization (a composite unfavorable outcome). Culture-negative status was defined as two negative-culture results at different visits without an intervening positive result. The date of culture-negative status was defined as the date of the first negative-culture result. This status continued until there were two positive cultures, without an intervening negative culture, or until there was a single positive culture that was not followed by two negative cultures. Relapse strains were those shown to be identical on 24-locus MIRU analysis.

The primary safety outcome was the proportion of patients with grade 3 or 4 adverse events that were graded according to a modified version of the toxicity criteria of the Division of AIDS of the National Institute of Allergy and Infectious Diseases.

\section{STATISTICAL ANALYSIS}

We determined that a sample size of 633 patients per group would provide a power of $85 \%$ to show noninferiority of the two moxifloxacin interventions to the control regimen with a margin of 6 percentage points, assuming a one-sided type I error of 0.0125 (Bonferroni correction). We estimated that $10 \%$ of the patients in each study group would have a unfavorable outcome and that $15 \%$ would have outcomes that could not be evaluated. (All definitions are provided in the Supplementary Appendix.) This margin of 6 percentage points reflected consultation with clinicians in high-burden countries and reanalysis of previous trials showing the effect of shortening treatment to 4 months without substituting a new drug.

Noninferiority was defined as a between-group 
difference of less than 6 percentage points in the upper boundary of the two-sided $97.5 \%$ Wald confidence interval for the proportion of patients with an unfavorable outcome. We used a generalized linear model with identity-link function with adjustment for stratification variables (weight group and study center). We performed both modified intention-to-treat and per-protocol analyses, with the latter considered to be the primary analysis. In the modified intention-to-treat analysis, we excluded patients with resistance to moxifloxacin or rifampin at baseline and those in whom the outcome could not be assessed (e.g., patients who had reinfection). (Detailed definitions are provided in Section 2 in the Supplementary Appendix.) We also performed a number of sensitivity and secondary analyses of the primary outcome to test the robustness of the results (Tables S3A and S3B in the Supplementary Appendix).

We used the chi-square test to compare the patients' sputum-culture status at the end of 8 weeks (intensive phase) across treatment groups and the log-rank test to compare the time to culture-negative status. We used similar methods to analyze other secondary outcomes, including the time to an unfavorable outcome, the status at the end of treatment, the status at 12 and 18 months among patients with a favorable outcome at end of treatment, and the status at 18 months according to a blinded clinical review of the data.

All patients who received at least one dose of a study medication were included in the safety analysis. The proportions of patients who had at least one grade 3 or 4 adverse event were compared across treatment groups with the use of the chi-square test.

\section{RESULTS}

\section{STUDY PATIENTS}

A total of 2763 patients were screened and 1931 underwent randomization: 909 in South Africa, 376 in India, 212 in Tanzania, 136 in Kenya, 119 in Thailand, 69 in Malaysia, 66 in Zambia, 22 in China, and 22 in Mexico (Table S4 in the Supplementary Appendix). The principal reasons for ineligibility were a lack of confirmation of smear positivity in the study laboratory, a CD4+ count of less than 250 cells per cubic millimeter, or multidrug-resistant disease, as detected by means of the Hain test (Fig. 1). The demographic and clinical characteristics of the patients were simi- lar in the three study groups (Table 1, and Tables S5 and S6 in the Supplementary Appendix).

The most common reason that patients were excluded from the modified intention-to-treat analysis was that they were found to be ineligible on the basis of data that were collected before randomization (e.g., lack of confirmation of the diagnosis of tuberculosis or confirmed multidrugresistant tuberculosis). The most common reasons for exclusion from the per-protocol analysis were a change of treatment for reasons other than treatment failure and a loss to follow-up (Fig. 1). Of the 1931 patients who underwent randomization, $89 \%$ in the isoniazid group, $92 \%$ in the ethambutol group, and $89 \%$ in the control group met the requirements for treatment adherence, which was based on receipt of approximately $80 \%$ of the assigned regimen (see the Supplementary Appendix for details).

\section{PRIMARY OUTCOME}

In the per-protocol analysis, a favorable outcome was reported in 436 patients (85\%) in the isoniazid group, as compared with 467 patients (92\%) in the control group, for an adjusted absolute difference of 6.1 percentage points $(97.5 \%$ confidence interval [CI], 1.7 to 10.5) favoring the control group (Table 2, and Fig. S2 in the Supplementary Appendix). A favorable outcome was reported in 419 patients $(80 \%)$ in the ethambutol group, for an adjusted absolute difference of 11.4 percentage points $(97.5 \%$ CI, 6.7 to 16.1$)$, as compared with the control group.

In the modified intention-to-treat analysis, the corresponding values also favored the control group, with a favorable outcome reported in 436 patients $(77 \%)$ in the isoniazid group, as compared with 468 (84\%) in the control group, for an adjusted absolute difference of 7.8 percentage points (97.5\% CI, 2.7 to 13.0), and in 419 patients (76\%) in the ethambutol group, for an adjusted absolute difference of 9.0 percentage points (97.5\% CI, 3.8 to 14.2) (Table 2, and Fig. S2 in the Supplementary Appendix). Results of all sensitivity analyses were consistent with those in the perprotocol and modified intention-to-treat analyses (Table S3A in the Supplementary Appendix).

The most common reason for an unfavorable outcome was relapse after conversion to culturenegative status after the end of active treatment (in 46 patients in the isoniazid group, 64 in the ethambutol group, and 12 patients in the control 


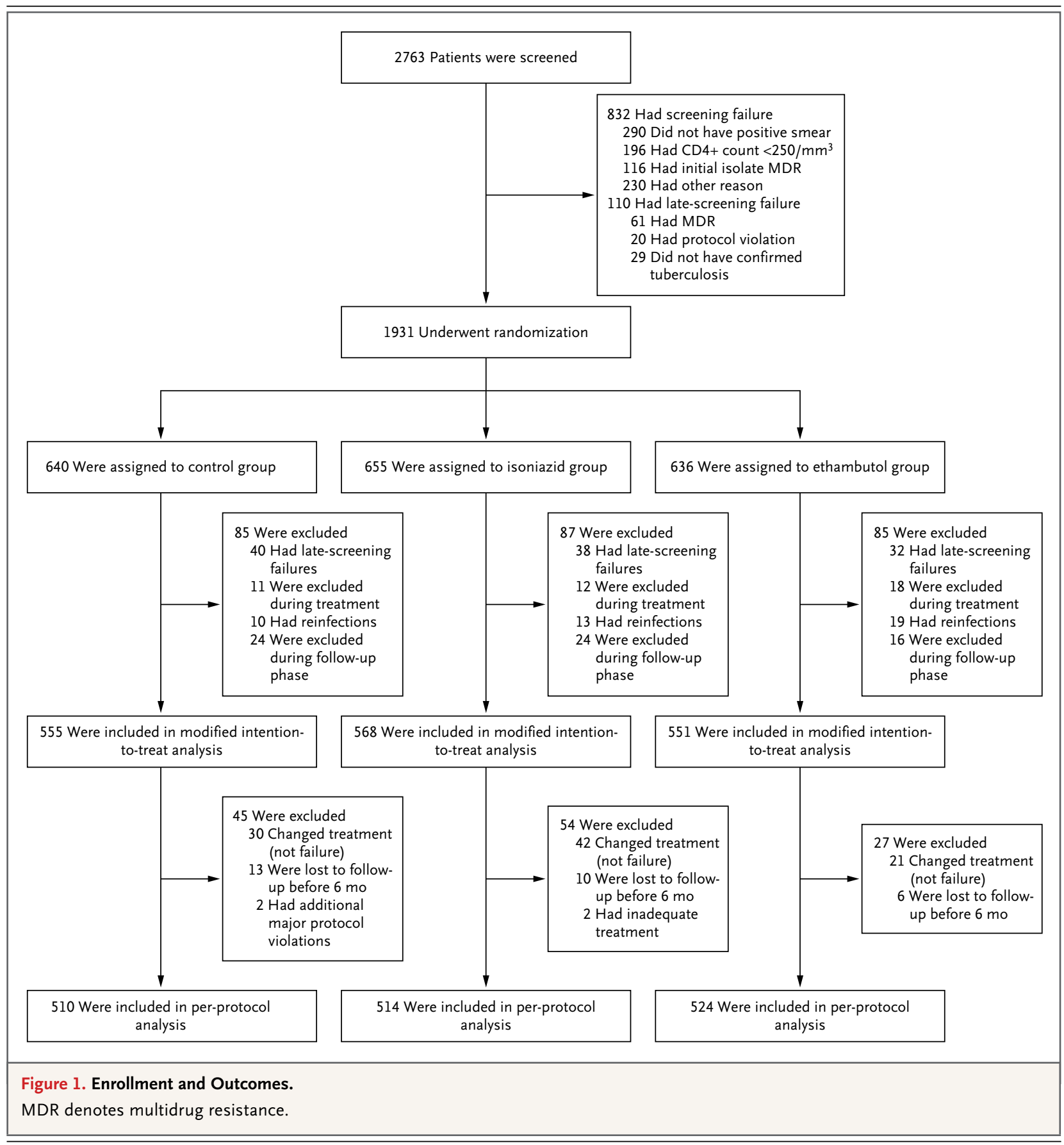

group). A similar pattern of results was seen in the modified intention-to-treat analysis (Table 2). There were no unequivocal cases of acquired resistance, but there were four cases of possible resistance - one in the ethambutol group (for moxifloxacin) and three in the control group (two for rifampin and one for isoniazid) - which require future whole-genome sequencing for interpretation.

\section{SUBGROUP ANALYSES}

There was no evidence that between-group differences in the primary outcome varied according to HIV status, region, recruitment site, age group, isoniazid susceptibility, or cavitation. The proportion of unfavorable outcomes among female patients, as compared with male patients, was similar in the three study groups (test of in- 


\begin{tabular}{|c|c|c|c|c|}
\hline Characteristic & $\begin{array}{l}\text { Control } \\
\text { Group } \\
(\mathrm{N}=510)\end{array}$ & $\begin{array}{l}\text { Isoniazid } \\
\text { Group } \\
(\mathrm{N}=514)\end{array}$ & $\begin{array}{c}\text { Ethambutol } \\
\text { Group } \\
(\mathrm{N}=524)\end{array}$ & $\begin{array}{l}\text { All Patients } \\
(\mathrm{N}=1548)\end{array}$ \\
\hline & \multicolumn{4}{|c|}{ number of patients (percent) } \\
\hline Male sex & $356(70)$ & $351(68)$ & $369(70)$ & $1076(70)$ \\
\hline \multicolumn{5}{|l|}{ Weight group广 } \\
\hline$<40 \mathrm{~kg}$ & $50(10)$ & $44(9)$ & $58(11)$ & $152(10)$ \\
\hline $40-45$ kg & 80 (16) & 90 (18) & $82(16)$ & 252 (16) \\
\hline$>45-55 \mathrm{~kg}$ & $206(40)$ & $210(41)$ & $204(39)$ & $620(40)$ \\
\hline$>55-75 \mathrm{~kg}$ & $161(32)$ & $158(31)$ & $174(33)$ & $493(32)$ \\
\hline$>75 \mathrm{~kg}$ & $13(3)$ & $12(2)$ & $6(1)$ & $31(2)$ \\
\hline \multicolumn{5}{|l|}{ Age group } \\
\hline$<25 \mathrm{yr}$ & $160(31)$ & $162(32)$ & $146(28)$ & $468(30)$ \\
\hline $25-35 \mathrm{yr}$ & $145(28)$ & $162(32)$ & $175(33)$ & $482(31)$ \\
\hline$>35 \mathrm{yr}$ & $205(40)$ & $190(37)$ & 203 (39) & 598 (39) \\
\hline \multicolumn{5}{|c|}{ Race or ethnic group $t$} \\
\hline Black & $238(47)$ & $210(41)$ & $237(45)$ & $685(44)$ \\
\hline Asian & $160(31)$ & $154(30)$ & $161(31)$ & $475(31)$ \\
\hline Mixed race & $111(22)$ & $148(29)$ & $126(24)$ & $385(25)$ \\
\hline Other & $1(<1)$ & $2(<1)$ & 0 & $3(<1)$ \\
\hline \multicolumn{5}{|l|}{ Smoking status } \\
\hline Never & $246(48)$ & $231(45)$ & $230(44)$ & $707(46)$ \\
\hline Past & $119(23)$ & $111(22)$ & $134(26)$ & $364(24)$ \\
\hline Current & $145(28)$ & $172(33)$ & $160(31)$ & $477(31)$ \\
\hline HIV positivity $\int$ & $38(7)$ & $37(7)$ & $35(7)$ & $110(7)$ \\
\hline \multicolumn{5}{|l|}{ Drug resistance } \\
\hline Isoniazid & $29(6)$ & $34(7)$ & $39(7)$ & $102(7)$ \\
\hline Pyrazinamide & $14(3)$ & $7(1)$ & $6(1)$ & $27(2)$ \\
\hline Cavitation\| & $368(72)$ & $357(69)$ & $367(70)$ & $1092(71)$ \\
\hline \multicolumn{5}{|c|}{ Time to positivity on MGIT sputum culture } \\
\hline$\geq 5$ days & $266(52)$ & $263(51)$ & $258(49)$ & $787(51)$ \\
\hline$<5$ days & $229(45)$ & $239(46)$ & $254(48)$ & $722(47)$ \\
\hline Not available & $15(3)$ & $12(2)$ & $12(2)$ & $39(3)$ \\
\hline \multicolumn{5}{|c|}{$\begin{array}{l}\text { There were no significant differences between the study groups. HIV denotes human immunodeficiency virus, and } \\
\text { MGIT Mycobacteria Growth Indicator Tube. } \\
\text { The median body-mass index (the weight in kilograms divided by the square of the height in meters) was } 18.4 \text { (range, } \\
12.1 \text { to } 50.9 \text { ) in the control group, } 18.3 \text { (range, } 12.0 \text { to } 33.1 \text { ) in the isoniazid group, } 18.4 \text { (range, } 12.2 \text { to } 32.6 \text { ) in the eth- } \\
\text { ambutol group, and } 18.3 \text { (range, } 12.0 \text { to } 50.9 \text { ) for all patients. } \\
\text { Race or ethnic group was reported by the investigator. Asian category included both South Asians and East Asians. } \\
\text { A single patient had missing HIV status. } \\
\text { Resistance results were missing for isoniazid in } 24 \text { patients and for pyrazinamide in } 27 \text { patients. } \\
\text { Cavitation status was missing for } 148 \text { patients. }\end{array}$} \\
\hline
\end{tabular}




\begin{tabular}{|c|c|c|c|c|c|c|c|c|}
\hline \multirow[t]{2}{*}{ Variable } & \multicolumn{4}{|c|}{ Per-Protocol Analysis } & \multicolumn{4}{|c|}{ Modified Intention-to-Treat Analysis } \\
\hline & $\begin{array}{l}\text { Control } \\
\text { Group } \\
(\mathrm{N}=510)\end{array}$ & $\begin{array}{l}\text { Isoniazid } \\
\text { Group } \\
(\mathrm{N}=514)\end{array}$ & $\begin{array}{l}\text { Ethambutol } \\
\text { Group } \\
(\mathrm{N}=524)\end{array}$ & $\begin{array}{c}\text { All } \\
\text { Patients } \\
(\mathrm{N}=1548)\end{array}$ & $\begin{array}{l}\text { Control } \\
\text { Group } \\
(\mathrm{N}=555)\end{array}$ & $\begin{array}{l}\text { Isoniazid } \\
\text { Group } \\
(\mathrm{N}=568)\end{array}$ & $\begin{array}{l}\text { Ethambutol } \\
\text { Group } \\
(\mathrm{N}=551)\end{array}$ & $\begin{array}{c}\text { All } \\
\text { Patients } \\
(\mathrm{N}=1674)\end{array}$ \\
\hline \multicolumn{9}{|l|}{ Favorable outcome - no. (\%) } \\
\hline Patients with outcome & $467(92)$ & $436(85)$ & $419(80)$ & $1322(85)$ & $468(84)$ & $436(77)$ & $419(76)$ & $1323(79)$ \\
\hline Culture-negative status at $18 \mathrm{mo}$ & $409(80)$ & $389(76)$ & $367(70)$ & $1165(75)$ & $410(74)$ & $389(68)$ & $367(67)$ & $1166(70)$ \\
\hline Unable to produce sputum & 0 & $2(<1)$ & 0 & $2(<1)$ & 0 & $2(<1)$ & 0 & $2(<1)$ \\
\hline $\begin{array}{l}\text { Unable to produce sputum at } \\
\qquad \begin{array}{l}18 \text { mo but culture- } \\
\text { negative status earlier }\end{array}\end{array}$ & $49(10)$ & $31(6)$ & $35(7)$ & $115(7)$ & $49(9)$ & $31(5)$ & $35(6)$ & $115(7)$ \\
\hline $\begin{array}{c}\text { Missing data on } L-J \text { culture at } \\
18 \text { mo and MGIT } \\
\text { negative }\end{array}$ & $9(2)$ & $14(3)$ & $17(3)$ & $40(3)$ & $9(2)$ & $14(2)$ & $17(3)$ & $40(2)$ \\
\hline \multicolumn{9}{|l|}{ Unfavorable outcome - no. (\%) } \\
\hline Patients with outcome & $43(8)$ & $78(15)$ & $105(20)$ & $226(15)$ & $87(16)$ & $132(23)$ & $132(24)$ & $351(21)$ \\
\hline \multicolumn{9}{|l|}{ 6-Mo treatment phase } \\
\hline Nonviolent death & $5(1)$ & $6(1)$ & $7(1)$ & $18(1)$ & $5(1)$ & $6(1)$ & $7(1)$ & $18(1)$ \\
\hline \multicolumn{9}{|l|}{ Treatment failure $\downarrow$} \\
\hline Culture-confirmed & $3(1)$ & $4(1)$ & $1(<1)$ & $8(1)$ & $3(1)$ & $4(1)$ & $1(<1)$ & $8(<1)$ \\
\hline Not culture-confirmed & $4(1)$ & $1(<1)$ & $4(1)$ & $9(1)$ & $4(1)$ & $1(<1)$ & $4(1)$ & $9(1)$ \\
\hline Adverse reaction & NA & NA & NA & NA & $18(3)$ & $15(3)$ & $9(2)$ & $42(3)$ \\
\hline Withdrawal of consent & NA & NA & NA & NA & $8(1)$ & $18(3)$ & $8(1)$ & $34(2)$ \\
\hline Relocation & NA & NA & NA & NA & $2(<1)$ & $4(1)$ & $4(1)$ & $10(1)$ \\
\hline Other investigator decision & NA & NA & NA & NA & $2(<1)$ & $5(1)$ & 0 & $7(<1)$ \\
\hline No completion of treatment & NA & NA & NA & NA & $13(2)$ & $10(2)$ & $6(1)$ & $29(2)$ \\
\hline \multicolumn{9}{|l|}{ Follow-up } \\
\hline $\begin{array}{l}\text { Relapse after culture-negative } \\
\text { status }\end{array}$ & $12(2)$ & $46(9)$ & $64(12)$ & $122(8)$ & $13(2)$ & $46(8)$ & $64(12)$ & $123(7)$ \\
\hline Retreated for tuberculosis & $14(3)$ & $17(3)$ & $27(5)$ & $58(4)$ & $14(3)$ & $18(3)$ & $27(5)$ & $59(4)$ \\
\hline $\begin{array}{c}\text { Death from tuberculosis or } \\
\text { respiratory distress }\end{array}$ & $2(<1)$ & 0 & 0 & $2(<1)$ & $2(<1)$ & 0 & 0 & $2(<1)$ \\
\hline \multicolumn{9}{|l|}{ No culture-negative status } \\
\hline Ever & $1(<1)$ & $1(<1)$ & 0 & $2(<1)$ & $1(<1)$ & $2(<1)$ & 0 & $3(<1)$ \\
\hline At last visit & $2(<1)$ & $3(1)$ & $2(<1)$ & $7(<1)$ & $2(<1)$ & $3(1)$ & $2(<1)$ & $7(<1)$ \\
\hline $\begin{array}{r}\text { Adjusted difference from control } \\
\text { in rate of unfavorable } \\
\text { outcome - percent- } \\
\text { age points }(97.5 \% \mathrm{CI})\end{array}$ & NA & $\begin{array}{c}6.1 \\
(1.7-10.5)\end{array}$ & $\begin{array}{c}11.4 \\
(6.7-16.1)\end{array}$ & NA & NA & $\begin{array}{c}7.8 \\
(2.7-13.0)\end{array}$ & $\begin{array}{c}9.0 \\
(3.8-14.2)\end{array}$ & NA \\
\hline
\end{tabular}

* The treatment phase was defined as any time from randomization to 32 weeks after randomization (26 weeks plus 6-week window). L-J denotes Lowenstein-Jensen solid medium, and NA not applicable.

$\dagger$ During follow-up, the relapse and retreatment categories include patients during the scheduled end of active treatment (after month 4 for the moxifloxacin-containing groups and month 6 for the control group). In the per-protocol analysis, data from 24-locus mycobacterial-interspersed-repetitive-unit analysis were missing for 9 of 17 patients with treatment failure, 42 of 122 patients with relapse, and 38 of 58 patients who were retreated for tuberculosis.

$\uparrow$ Listed are patients who were receiving active treatment in whom treatment failed.

The New England Journal of Medicine 


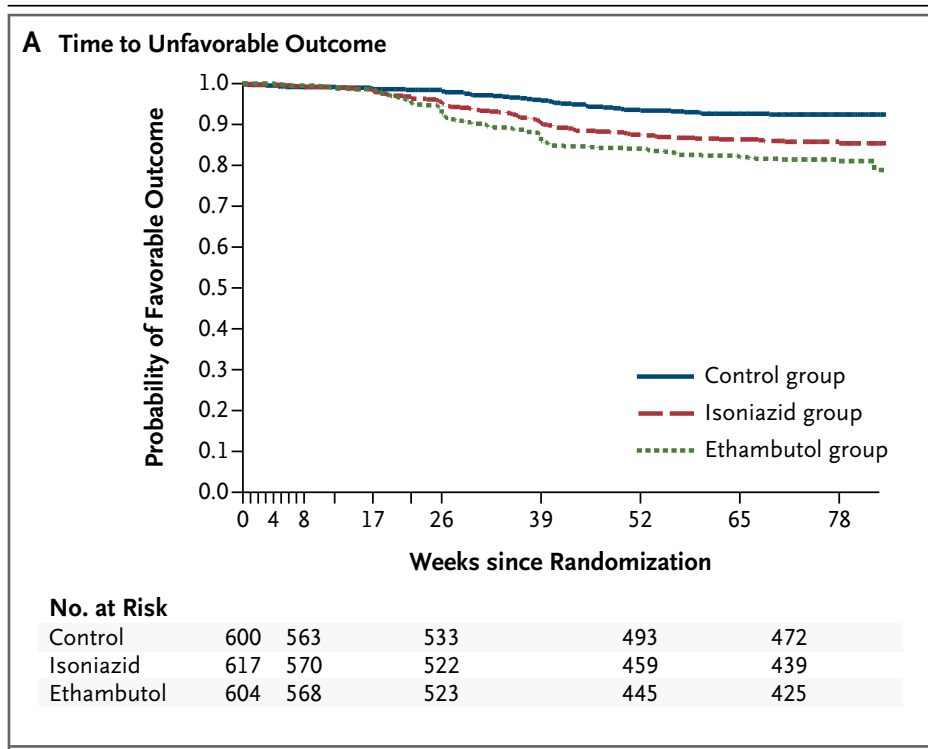

B Time to Culture-Negative Status

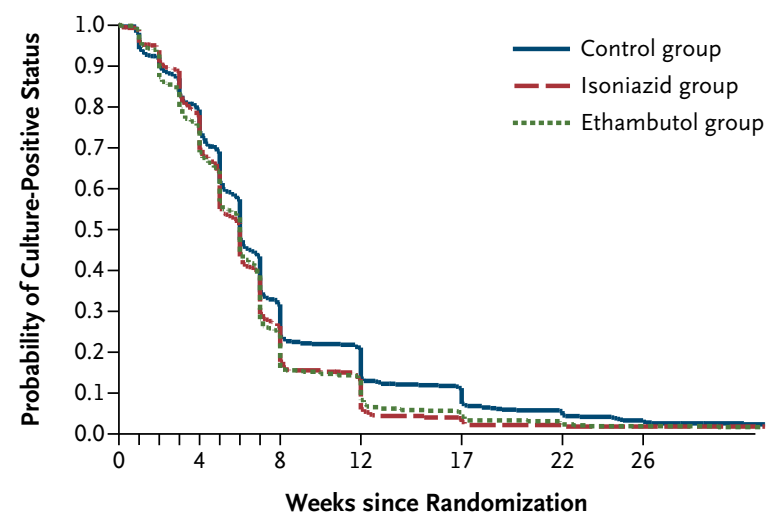

No. at Risk

$\begin{array}{lrrrrrr}\text { Control } & 600 & 465 & 183 & 122 & 64 & 19 \\ \text { Isoniazid } & 617 & 459 & 154 & 76 & 21 & 9 \\ \text { Ethambutol } & 604 & 449 & 141 & 79 & 30 & 9\end{array}$

Figure 2. Kaplan-Meier Estimates of the Time to an Unfavorable Outcome and Conversion to Culture-Negative Status.

Panel A shows that the time until patients had an unfavorable outcome was shorter in the isoniazid group than in the control group (hazard ratio, 1.25 [97.5\% Cl, 1.08 to 1.42$]$ ) and was further reduced in the ethambutol group (hazard ratio, 1.21 [97.5\% Cl, 1.05 to 1.37$]$ ). Panel B shows the time until conversion to culture-negative status, which occurred sooner in the isoniazid group and the ethambutol group than in the control group, according to analyses of sputum samples cultured in Lowenstein-Jensen solid medium. Patients who were excluded from the primary per-protocol analysis were included in this analysis, but data were censored at the time of exclusion from the per-protocol analysis.

teraction, $\mathrm{P}=0.004$ for the isoniazid group and $\mathrm{P}=0.02$ for the ethambutol group) (Table S3B in the Supplementary Appendix).

\section{TIME TO NEGATIVE-CULTURE STATUS}

In Kaplan-Meier analyses, patients in the isoniazid group and the ethambutol group had conversion to culture-negative status sooner than those in the control group in sputum analyses with the use of Lowenstein-Jensen solid medium (Fig. 2B) and MGIT medium (Fig. S3 and Table S7 in the Supplementary Appendix) ( $\mathrm{P}<0.01$ for both analyses). More patients receiving the moxifloxacincontaining regimens had culture-negative status at 8 weeks, but the difference was not significant (Table S8 in the Supplementary Appendix).

\section{TIME TO AN UNFAVORABLE OUTCOME}

In the per-protocol analyses, the time to an unfavorable outcome was shorter in the isoniazid group than in the control group (hazard ratio, 1.87; $97.5 \%$ CI, 1.07 to 2.67 ) and was further reduced in the ethambutol group (hazard ratio, 2.56; 97.5\% CI, 1.51 to 3.60) (Fig. 2A, and Table S9 in the Supplementary Appendix).

\section{ADVERSE EVENTS}

There were no significant between-group differences in the incidence of grade 3 or 4 adverse events, with reports of events in 127 patients (19\%) in the isoniazid group and 111 patients (17\%) in the ethambutol group, as compared with $123 \mathrm{pa}$ tients (19\%) in the control group (Table 3). A total of 349 serious adverse events occurred in 173 patients, with 246 events occurring during the treatment period and 103 during follow-up. There were 43 deaths ( 16 during the treatment period and 27 during follow-up) during the study, 30 of which were deemed to be tuberculosis-related (Table S10 in the Supplementary Appendix). Overall, the numbers of serious adverse events, types of events, and numbers of patients with events (including the number of deaths) were similar in the three study groups during both the treatment period and the follow-up period.

There were no significant between-group differences in the incidence of adverse events of special interest, including tendinopathy, seizure, clinically significant cardiac toxicity, hypoglycemia or hyperglycemia, and peripheral neuropathy. The proportions of events were similar in the study groups when all adverse events were considered. There were no significant differences in any measures of biochemical, hematologic, or hepatic safety. 


\begin{tabular}{|c|c|c|c|c|}
\hline \multicolumn{5}{|l|}{ Table 3. Safety Analysis.* } \\
\hline \multirow[t]{2}{*}{ Adverse Event } & $\begin{array}{l}\text { Control Group } \\
(N=639)\end{array}$ & $\begin{array}{l}\text { Isoniazid Group } \\
\quad(\mathrm{N}=655)\end{array}$ & $\begin{array}{l}\text { Ethambutol Group } \\
\qquad(N=636)\end{array}$ & $\begin{array}{c}\text { All Patients } \\
(\mathrm{N}=1930)\end{array}$ \\
\hline & \multicolumn{4}{|c|}{ number of patients (percent) } \\
\hline \multicolumn{5}{|l|}{$\begin{array}{l}\text { During treatment phase } \\
\text { or follow-up }\end{array}$} \\
\hline Any & $123(19)$ & $127(19)$ & $111(17)$ & 361 (19) \\
\hline Grade 3 only & $83(13)$ & $90(14)$ & $82(13)$ & $255(13)$ \\
\hline Grade 4 & $40(6)$ & $37(6)$ & $29(5)$ & $106(5)$ \\
\hline Serious adverse event & $59(9)$ & $62(9)$ & $52(8)$ & $173(9)$ \\
\hline \multicolumn{5}{|l|}{ Death } \\
\hline Any & $16(3)$ & $15(2)$ & $12(2)$ & $43(2)$ \\
\hline Tuberculosis-related & $11(2)$ & $10(2)$ & $9(1)$ & $30(2)$ \\
\hline \multicolumn{5}{|c|}{ During treatment phase only } \\
\hline Any & $111(17)$ & $105(16)$ & $99(16)$ & $315(16)$ \\
\hline Grade 3 only & $76(12)$ & $71(11)$ & $73(11)$ & $220(11)$ \\
\hline Grade 4 & $35(5)$ & $34(5)$ & $26(4)$ & $95(5)$ \\
\hline Serious adverse event & $46(7)$ & $40(6)$ & $35(6)$ & $121(6)$ \\
\hline \multicolumn{5}{|l|}{ Death } \\
\hline Any & $5(1)$ & $6(1)$ & $5(1)$ & $16(1)$ \\
\hline Tuberculosis-related & $4(1)$ & $6(1)$ & $5(1)$ & $15(1)$ \\
\hline
\end{tabular}

\section{DISCUSSION}

In this phase 3 trial, we aimed to determine whether the promising data that were observed for moxifloxacin in studies in animals and phase 2 studies translated into an effective reduction in the duration of the standard tuberculosis treatment regimen. The trial showed that the substitution of moxifloxacin in 4-month regimens based on either isoniazid or ethambutol did not meet the margin for noninferiority, as compared with the 6-month control regimen. The same conclusions were reached when the outcome was determined with the use of MGIT cultures of sputum samples. Among patients receiving the two moxifloxacin-containing regimens, a small number had treatment failures, but a larger number had a relapse after the end of active treatment. The difference between the isoniazid group and the ethambutol group may be due to the bactericidal effect of isoniazid or the presence of three drugs over a 4-month period. The similarity in outcome among women in the isoniazid group and the control group may represent a chance finding but merits further investigation.

It has been previously suggested that Asian patients often have a more chronic form of tuberculosis with a different clinical course than that in African patients, ${ }^{18,19}$ but we did not see any evidence of variation in clinical-disease outcome in the different racial groups. Our approach in the conduct of this trial, including standardized laboratory methods and clinical management, has resulted in consistent results across more than 20 sensitivity analyses, with minimal variation among study centers on different continents.

In comparison with other trials that used fluoroquinolones in a 4-month regimen, the rates of an unfavorable outcome in the experimental groups in our study are lower than those in the RIFAQUIN regimen ${ }^{20}$ and similar to those found 
in the OFLOTUB trial. ${ }^{21}$ In trials evaluating 4-month streptomycin-containing regimens that were performed in the 1970s in East Africa and Singapore, rates of relapse ranged from 11 to $40 \%$ after 2 years of follow-up. ${ }^{18,19}$

In our study, a daily regimen of moxifloxacin in combination with standard antituberculosis agents for 4 months had an acceptable side-effect profile. We did not find any evidence of either hypoglycemia or hyperglycemia or tendinopathies that have been associated with fluoroquinolones, ${ }^{22,23}$ nor did we find evidence of increased hepatic dysfunction, a potential concern in regimens containing moxifloxacin or lacking isoniazid. ${ }^{24}$ There was no clinical evidence of cardiac toxicity, although electrocardiography was not performed systematically. These are important findings for future regimens that may use moxifloxacin in combination with other agents in tuberculosis treatment. ${ }^{25}$

Our findings raise questions about progression decisions throughout the development pathway for tuberculosis drugs. Data from studies in mice predicted that the inclusion of moxifloxacin would result in a reduction of 1 to 2 months in the treatment duration, as compared with standard therapy. ${ }^{12,13}$ In our study of such treatment shortening, the moxifloxacin-containing regimens did not work adequately, suggesting that the murine model may have overpredicted the sterilizing potency of moxifloxacin in this regimen.

More important is the observed poor predictability of culture conversion for long-term outcomes. Although 2-month culture conversion is associated with relapse-free cure, this observed correlation in populations is not strong enough to reliably predict outcomes for individual patients or definitively guide the selection of regimen in drug development. ${ }^{26,27}$ This finding underlines the importance of the content and duration of treatment in the following weeks. ${ }^{28}$ Four 2-month studies of the inclusion of moxifloxacin in the standard regimen have been reported, with variable results. $^{14-16,29}$ The only study to report a hazard ratio for the time to culture conversion was that of Rustomjee et al., ${ }^{14}$ who, in a study involving approximately 50 patients per group, found that the hazard ratio for the time to culture conversion for the moxifloxacin-containing regimen, as compared with the standard regimen, was 1.73, indicating a shorter duration. This raised the pos- sibility that a 4-month regimen might be effective, although the $95 \%$ confidence interval ranged from 1.15 to 2.60. In our study, with more than 600 patients in each group, we found a more precise estimate of the hazard ratio to be 1.25 ( $95 \%$ CI, 1.10 to 1.40), a result that is within the confidence interval found previously ${ }^{14}$ but with a smaller effect, which would seem unlikely to merit progression to a phase 3 trial. Thus, such short trials may correlate with long-term outcomes, but the small sample size and resulting wide confidence intervals limit their ability to predict treatment shortening.

This limitation suggests that efficient drug development for tuberculosis may require a different approach. Instead of relying on the results of 2-month phase 2 trials to select candidate regimens for phase 3 studies, investigators might find that the most efficient approach is to conduct phase 3 trials as quickly as possible while establishing more feasible and less costly approaches to performing these studies. Possible improvements could include larger noninferiority margins, permitting smaller sample sizes, and building multiple treatment durations into each study.

In conclusion, in patients with uncomplicated, smear-positive tuberculosis, the noninferiority of the moxifloxacin-containing regimens was not shown, despite the fact that these regimens had better bactericidal activity than the standard control regimen. ${ }^{30}$

Supported by the Global Alliance for TB Drug Development with support from the Bill and Melinda Gates Foundation, the European and Developing Countries Clinical Trials Partnership, U.S. Agency for International Development, U.K. Department for International Development, Directorate General for International Cooperation of the Netherlands, Irish Aid, Australia Department of Foreign Affairs and Trade, and National Institutes of Health, AIDS Clinical Trials Group and by grants from the National Institute of Allergy and Infectious Diseases (NIAID) (UM1AI068634, UM1 AI068636, and UM1AI106701) and by NIAID grants to the University of KwaZulu Natal, South Africa, AIDS Clinical Trials Group (ACTG) site 31422 (1U01AI069469); to the Perinatal HIV Research Unit, Chris Hani Baragwanath Hospital, South Africa, ACTG site 12301 (1U01AI069453); and to the Durban International Clinical Trials Unit, South Africa, ACTG site 11201 (1U01AI069426); Bayer Healthcare for the donation of moxifloxacin; and Sanofi for the donation of rifampin.

Disclosure forms provided by the authors are available with the full text of this article at NEJM.org.

We thank all the patients for their participation in the study; the nursing and laboratory staff; all those who advised, volunteered, or otherwise supported community engagement around the REMoxTB clinical trial sites; and Sangita Patel, Marie Messina, Melanie Barry, Derek Ambrosino, and Joanna Breitstein for providing administrative support. 


\section{REFERENCES}

1. Gillespie SH, Billington O. Activity of moxifloxacin against mycobacteria. J Antimicrob Chemother 1999;44:393-5.

2. Falzon D, Jaramillo E, Schünemann HJ, et al. WHO guidelines for the programmatic management of drug-resistant tuberculosis: 2011 update. Eur Respir J 2011; 38:516-28.

3. Johnston JC, Shahidi NC, Sadatsafavi M, Fitzgerald JM. Treatment outcomes of multidrug-resistant tuberculosis: a systematic review and meta-analysis. PLoS One 2009;4(9):e6914.

4. Ginsberg AM. Tuberculosis drug development: progress, challenges, and the road ahead. Tuberculosis (Edinb) 2010;90 162-7.

5. Grossman RF, Hsueh P-R, Gillespie $\mathrm{SH}$, Blasi F. Community-acquired pneumonia and tuberculosis: differential diagnosis and the use of fluoroquinolones. Int J Infect Dis 2014;18:14-21.

6. Müller M, Stass H, Brunner M, Möller JG, Lackner E, Eichler HG. Penetration of moxifloxacin into peripheral compartments in humans. Antimicrob Agents Chemother 1999;43:2345-9.

7. Lubasch A, Keller I, Borner K, Koeppe P, Lode H. Comparative pharmacokinetics of ciprofloxacin, gatifloxacin, grepafloxacin, levofloxacin, trovafloxacin, and moxifloxacin after single oral administration in healthy volunteers. Antimicrob Agents Chemother 2000;44:2600-3.

8. Sullivan JT, Woodruff M, Lettieri J, et al. Pharmacokinetics of a once-daily oral dose of moxifloxacin (Bay 12-8039), a new enantiomerically pure 8-methoxy quinolone. Antimicrob Agents Chemother 1999; 43:2793-7.

9. Miyazaki E, Miyazaki M, Chen JM, Chaisson RE, Bishai WR. Moxifloxacin (BAY12-8039), a new 8-methoxyquinolone, is active in a mouse model of tuberculosis. Antimicrob Agents Chemother 1999;43: 85-9.

10. Gosling RD, Uiso LO, Sam NE, et al. The bactericidal activity of moxifloxacin in patients with pulmonary tuberculosis. Am J Respir Crit Care Med 2003;168:13425.

11. Pletz MWR, De Roux A, Roth A, Neumann $\mathrm{K}-\mathrm{H}$, Mauch H, Lode H. Early bactericidal activity of moxifloxacin in treatment of pulmonary tuberculosis: a prospective, randomized study. Antimicrob Agents Chemother 2004;48:780-2.

12. Nuermberger EL, Yoshimatsu T, Tyagi $S$, et al. Moxifloxacin-containing regimen greatly reduces time to culture conversion in murine tuberculosis. Am J Respir Crit Care Med 2004;169:421-6.

13. Nuermberger EL, Yoshimatsu T, Tyagi $\mathrm{S}$, et al. Moxifloxacin-containing regimens of reduced duration produce a stable cure in murine tuberculosis. Am J Respir Crit Care Med 2004;170:1131-4.

14. Rustomjee R, Lienhardt C, Kanyok T, et al. A phase II study of the sterilising activities of ofloxacin, gatifloxacin and moxifloxacin in pulmonary tuberculosis. Int J Tuberc Lung Dis 2008;12:128-38.

15. Conde $\mathrm{MB}$, Efron A, Loredo $\mathrm{C}$, et al. Moxifloxacin versus ethambutol in the initial treatment of tuberculosis: a doubleblind, randomised, controlled phase II trial. Lancet 2009;373:1183-9.

16. Dorman SE, Johnson JL, Goldberg S, et al. Substitution of moxifloxacin for isoniazid during intensive phase treatment of pulmonary tuberculosis. Am J Respir Crit Care Med 2009;180:273-80.

17. Bryant JM, Harris SR, Parkhill J, et al. Whole-genome sequencing to establish relapse or re-infection with Mycobacterium tuberculosis: a retrospective observational study. Lancet Respir Med 2013;1:786-92.

18. East African/British Medical Research Council. Controlled clinical trial of five short-course (4-month) chemotherapy regimens in pulmonary tuberculosis: second report of the 4th study. Am Rev Respir Dis 1981;123:165-70.

19. Singapore Tuberculosis Service/British Medical Research Council. Clinical trial of six-month and four-month regimens of chemotherapy in the treatment of pulmonary tuberculosis: the results up to 30 months. Tubercle 1981;62:95-102.

20. Jindani A. Recent progress in TB clinical trials: results of the RIFAQUIN trial. Presented at the 41st World Conference on Lung Health of the International Union Against Tuberculosis and Lung Disease, Paris, October 30-November 3, 2013. abstract.

21. Merle CS, Fielding KL, Lapujade O, et al. 4-Month regimen for treating drug- susceptible pulmonary tuberculosis: main efficacy and safety results of the OFLOTUB Trial. Presented at the 41st World Conference on Lung Health of the International Union Against Tuberculosis and Lung Disease, Paris, October 30-November 3, 2013. abstract.

22. Park-Wyllie LY, Juurlink DN, Kopp A, et al. Outpatient gatifloxacin therapy and dysglycemia in older adults. N Engl J Med 2006;354:1352-61.

23. Khaliq Y, Zhanel GG. Fluoroquinolone-associated tendinopathy: a critical review of the literature. Clin Infect Dis 2003;36:1404-10.

24. Update: fatal and severe liver injuries associated with rifampin and pyrazinamide for latent tuberculosis infection, and revisions in American Thoracic Society/CDC recommendations - United States, 2001. MMWR Morb Mortal Wkly Rep 2001;50:733-5.

25. Diacon AH, Dawson R, von GrooteBidlingmaier F, et al. 14-Day bactericidal activity of PA-824, bedaquiline, pyrazinamide, and moxifloxacin combinations: a randomised trial. Lancet 2012;380:98693.

26. Aber VR, Nunn AJ. Short term chemotherapy of tuberculosis: factors affecting relapse following short term chemotherapy. Bull Int Union Tuberc 1978;53:276-80. (In French.)

27. Phillips PPJ, Fielding K, Nunn AJ. An evaluation of culture results during treatment for tuberculosis as surrogate endpoints for treatment failure and relapse. PLoS One 2013;8(5):e63840.

28. Nunn AJ, Jindani A, Enarson DA. Results at 30 months of a randomised trial of two 8-month regimens for the treatment of tuberculosis. Int J Tuberc Lung Dis 2011;15:741-5.

29. Burman WJ, Goldberg S, Johnson JL, et al. Moxifloxacin versus ethambutol in the first 2 months of treatment for pulmonary tuberculosis. Am J Respir Crit Care Med 2006;174:331-8.

30. Nunn AJ, Phillips PPJ, Mitchison DA. Timing of relapse in short-course chemotherapy trials for tuberculosis. Int J Tuberc Lung Dis 2010;14:241-2.

Copyright (c) 2014 Massachusetts Medical Society. 